\title{
Foliar Application of Dikegulac Sodium Increases Branching of 'Merritt's Supreme' Bigleaf Hydrangea
}

\author{
Youping Sun ${ }^{1}$, Guihong $\mathrm{Bi}^{2}$, Genhua $\mathrm{Niu}^{1,4}$, and Christina Perez ${ }^{3}$
}

AdDitional Index wORDs. Hydrangea macrophylla 'Merritt's Supreme', lateral branching, phytotoxicity, pinching, plant growth regulator

SUMMARY. The goal of this experiment was to evaluate the efficiency of foliar application of dikegulac sodium on increasing the lateral branching of 'Merritt's Supreme' bigleaf hydrangea (Hydrangea macrophylla). Plants were grown in greenhouses at two locations including El Paso, TX and Kosciusko, MS. Two weeks before application of dikegulac sodium, half of plants were hand-pinched leaving two nodes. Foliar spray of dikegulac sodium at 400,800 , or $1600 \mathrm{mg} \cdot \mathrm{L}^{-1}$ was then applied to pinched and unpinched plants. There were two additional control treatments: pinched or unpinched without application of dikegulac sodium. Data were collected at 2 weeks, 6 weeks, 80 days, and 10 months after treatments. Bigleaf hydrangea plants exhibited severe phytotoxicity including interveinal chlorosis or bleaching of new growth at 2 weeks after application of dikegulac sodium with more pronounced symptoms at higher dikegulac sodium concentrations. The severity of phytotoxicity symptoms became less significant at 6 weeks after treatment. The effect of dikegulac sodium on bigleaf hydrangea plant growth, number of branches, and number of flowers depended on both locations and dosages. In El Paso, TX, dikegulac sodium at 800 or $1600 \mathrm{mg} \cdot \mathrm{L}^{-1}$ inhibited bigleaf hydrangea plant growth at 6 weeks and 80 days after treatment, and this effect disappeared at 10 months after treatment. Dikegulac sodium at all tested dosages doubled or tripled the number of branches of pinched or unpinched bigleaf hydrangea, respectively, at 80 days after treatment. At 10 months after treatment, the number of branches and flowers of bigleaf hydrangea plants tended to increase, but was insignificant. In Kosciusko, MS, dikegulac sodium at $1600 \mathrm{mg} \cdot \mathrm{L}^{-1}$ reduced the plant growth at 6 weeks after treatment. This treatment increased the number of branches and flowers of unpinched plants by $196 \%$ and $95 \%$ and pinched plants by $53 \%$ and $31 \%$, respectively, at 10 months after treatment. Dikegulac sodium application could be used to increase number of branches and flowers and produce compact 'Merritt's Supreme' bigleaf hydrangea. However, the efficacy varied with environmental conditions.

$\mathrm{H}$ ydrangeas (Hydrangea sp.) are popular ornamental plants due to their enormous, round flower heads blooming in white, pink, red, purple, or blue in spring and summer. It was listed as

This project was supported by the USDA National Institute of Food and Agriculture, Hatch Projects TEX090450 and MIS212050 and The IR-4 Project. We also gratefully acknowledge financial support from the National Institute of General Medical Sciences of the National Institutes of Health under award no. R25GM060424. Mention of a trademark, proprietary product, or vendor does not constitute a guarantee or warrantee of the product and does not imply its approval to the exclusion of other products or vendors that also may be suitable. The authors thank Oregon Hydrangea Company for donating cuttings and Mark Terkanian from Natchez Trace Greenhouse for his technical support.

${ }^{1}$ Texas A\&M AgriLife Research and Extension Center at El Paso, Texas A\&M University System, El Paso, TX 79927

${ }^{2}$ Department of Plant and Soil Sciences, Mississippi State University, Mississippi State, MS 39762

${ }^{3}$ El Paso Community College, El Paso, TX 79925

${ }^{4}$ Corresponding author. E-mail: gniu@ag.tamu.edu. the third most used plant in a 2011 survey of 4000 landscape professionals (GIE Media, 2011). There are at least 70 hydrangea species native to southern and eastern Asia and the Americas (Wikipedia, 2014). In the United States, only five hydrangea species are widely cultivated (U.S. National Arboretum, 2005), and an estimated $\$ 73$ million hydrangea plants were sold annually (U.S. Department of Agriculture, 2009).
Bigleaf hydrangea is one of the most widely grown hydrangea species in the United States with over 1000 cultivars (Dirr, 2012). Some cultivars are best pruned or pinched on an annual basis to prevent them from being "leggy" and to promote lateral branching.

Pruning or pinching is a common technique in hydrangea production to reduce plant size, stimulate lateral branching, improve overall appearance, and enhance flower development. But hand pruning or pinching is labor intensive and costly. If done improperly, it can also reduce flowering in hydrangea plants (U.S. National Arboretum, 2005). Alternatively, a wide range of plant growth regulators (PGRs) with diverse modes of action, sites of absorption and activity are available to control height and promote lateral branching. Their use varies with plant species, chemical concentration, time of application, and environmental conditions. For example, ethephon has been reported to effectively reduce the height of 'Sonora Jingle' poinsettia (Euphorbia pulcherrima) by $33 \%$ and 'Sonora White' poinsettia by $31 \%$ when 700 $\mathrm{mg} \cdot \mathrm{L}^{-1}$ ethephon was sprayed three times on 29 Aug., 20 Sept., and 13 Oct. 2005, respectively (Sun et al., 2011). Ethephon acts via release of ethylene, which is absorbed by the plant and causes cells to limit elongation and increase in width (Taiz and Zeiger, 2010). Unlike ethephon, most PGRs inhibit the biosynthesis of gibberellins within plants and therefore function as antigibberellins to suppress stem elongation (Bailey and Whipker, 1998). For example, seashore mallow (Kosteletzkya virginica) plants treated with chlormequat chloride at 750 , 1000 , or $1500 \mathrm{mg} \cdot \mathrm{L}^{-1}$ or paclobutrazol at $60 \mathrm{mg} \cdot \mathrm{L}^{-1}$ were effective for height control and lateral branching (Hilgers et al., 2005).

\begin{tabular}{llll}
\hline $\begin{array}{l}\text { Units } \\
\text { To convert U.S. to SI, } \\
\text { multiply by }\end{array}$ & U.S. unit & SI unit & $\begin{array}{l}\text { To convert SI to U.S., } \\
\text { multiply by }\end{array}$ \\
\hline 10 & $\%$ & $\mathrm{~g} \cdot \mathrm{L}^{-1}$ & 0.1 \\
29.5735 & $\mathrm{fl} \mathrm{oz}$ & $\mathrm{mL}$ & 0.0338 \\
7.8125 & $\mathrm{fl} \mathrm{oz} / \mathrm{gal}$ & $\mathrm{mL} \cdot \mathrm{L}^{-1}$ & 0.1280 \\
2.54 & inch $(\mathrm{es})$ & $\mathrm{cm}$ & 0.3937 \\
16.3871 & inch & $\mathrm{cm}^{3}$ & 0.0610 \\
1 & $\mathrm{mmho} / \mathrm{cm}$ & $\mathrm{dS} \cdot \mathrm{m}^{-1}$ & 1 \\
28.3495 & $\mathrm{oz}$ & $\mathrm{g}$ & 0.0353 \\
1 & $\mathrm{ppm}$ & $\mathrm{mg} \cdot \mathrm{L}^{-1}$ & 1 \\
$\left({ }^{\circ} \mathrm{F}-32\right) \div 1.8$ & ${ }^{\circ} \mathrm{F}$ & ${ }^{\circ} \mathrm{C}$ & $\left({ }^{\circ} \mathrm{C} \times 1.8\right)+32$
\end{tabular}


Symptoms of phytotoxicity are a known issue with most plant growth retardants. The severity of phytotoxicity depends on plant species or cultivar and the chemical concentration. Foliar application of ethephon to 'Sonora Jingle' and 'Sonora White' poinsettia exhibited phytotoxicity as indicated by reduced biomass accumulation (Sun et al., 2011). Leaf chlorosis appeared on seashore mallow plants immediately after application of paclobutrazol, chlormequat chloride, and chlormequat chloride/ daminozide, and disappeared 8 weeks after treatments (Hilgers et al., 2005). This phytotoxicity would reduce plant marketability and should be avoided by choosing an optimized concentration of PGR for producing compact plants.

Dikegulac sodium $[18.5 \%$ dikegulac sodium (Augeo ${ }^{\circledR}$; OHP, Mainland, PA)] is a PGR labeled to reduce or break apical dominance in plant shoots and to enhance lateral branching by disrupting cell wall integrity. Dikegulac sodium could be used to produce more compact plants with an increased number of flowers. It can also be used to enhance the overall structure and appearance of ornamentals including bedding plants, herbaceous plants, perennials, woody ornamentals and trees. Dikegulac sodium sprayed between 4000 and $6000 \mathrm{mg} \cdot \mathrm{L}^{-1}$ to mock orange (Murraya paniculata) hedge decreased apical dominance among lateral shoots and enhanced uniform regrowth without causing visible damage (Kawabata and Criley, 1996). Norcini et al. (1993) recommended that a foliar spray of dikegulac sodium at $1600 \mathrm{mg} \cdot \mathrm{L}^{-1}$ could potentially reduce the amount of pruning required during 'Barbara Karst' bougainvillea [Bougainvillea $\times$ buttiana (B. glabra $\times$ B. peruviana)] production. However, many factors including species, ingredient dosage, cultural and environmental conditions affect the efficacy of dikegulac sodium on plant growth and development. For example, foliar application of dikegulac sodium at 500 , 1000 , or $1500 \mathrm{mg} \cdot \mathrm{L}^{-1}$ did not control the height and increase the number of branches of seashore mallow (Hilgers et al., 2005). However, Grossman et al. (2013) reported that the application of dikegulac sodium to herbaceous perennial liners ['Autumn Joy' butterfly stonecrop (Sedum spectabile), 'Bright Eyes' garden phlox (Pblox paniculata), 'Walker's Low' dwarf catmint (Nepeta racemosa)] before transplanting increased branching, with higher rates of dikegulac sodium being more effective in liners. Carter et al. (1996) observed that dikegulac sodium at 250,500 , or $750 \mathrm{mg} \cdot \mathrm{L}^{-1}$ increased shoot number without decreasing frond length of boston fern (Nephrolepsis exaltata). Al-Juboory and Williams (1991) observed that dikegulac sodium not only stimulated branching, but also reduced branch length in golden pothos (Scindapsus aureus). Dikegulac sodium has also been reported to reduce the stem length of 'Enchantment' and 'Joan Evans' midcentury hybrid lily (Lilium $\times$ bybrida), but prevent the development of flower buds and plant senescence (Hanks and Menhenett, 1980). Norcini et al. (1993) observed that dikegulac sodium stimulated profuse flowering of both 'Barbara Karst' and 'Rainbow Gold' bougainvillea during middle spring to early summer. Symptoms of phytotoxicity such as yellow and narrow leaves were also present in finished plants of 'Autumn Joy' butterfly stonecrop, 'Bright Eyes' garden phlox, 'Walker's Low' dwarf catmint, 'Moonshine' yarrow (Achillea filipendulina) that were treated with $1600 \mathrm{mg} \cdot \mathrm{L}^{-1}$ dikegulac sodium (Grossman et al., 2013).

Dikegulac sodium has been used to successfully control height of Little Lime $^{\mathrm{TM}}$ hardy hydrangea $[H$. paniculata 'Jane' (Cochran and Fulcher, 2013)] and 'Limelight' hardy hydrangea (Cochran et al., 2013). Both hydrangea cultivars set flower buds on current season wood. However, 'Merritt's Supreme' bigleaf hydrangea produces blooms on old wood. Therefore, the timing of foliar application and dosage of dikegulac sodium might be different for hardy hydrangea and bigleaf hydrangea. The objectives of this project were to investigate the efficacy of dikegulac sodium on lateral branching applied as a foliar spray and possible phytotoxicity on 'Merritt's Supreme' bigleaf hydrangea in two greenhouse experiments at two locations, El Paso, TX, and Kosciusko, MS.

\section{Materials and methods}

Plant materials, Culture, AND TREATMENTS. The study was conducted at two locations: Texas AgriLife Research Center at El Paso, TX (lat. $31^{\circ} 41^{\prime} 47.31^{\prime \prime} \mathrm{N}$, long. $106^{\circ} 16^{\prime} 55.07^{\prime \prime} \mathrm{W}$ ) and Natchez Trace Greenhouse, Kosciusko, MS (lat. 3302'27.39"N, long. $\left.89^{\circ} 34^{\prime} 53.54^{\prime \prime} \mathrm{W}\right)$. Softwood cuttings of 'Merritt's Supreme' bigleaf hydrangea were received on 3 May 2012 from Oregon Hydrangea Company (Brookings, OR). At both locations, bigleaf hydrangea cuttings were treated as quick-dip with $1000 \mathrm{mg} \cdot \mathrm{L}^{-1}$ indole-3-butyric acid (IBA) [1.0\% IBA, 0.5\% 1-napthaleneacetic acid, 98.5\% inert ingredients (Dip'N Grow ${ }^{\circledR}$ liquid rooting concentrate; Dip'N Grow ${ }^{\circledR}$, Clackamas, OR)]. All cuttings were stuck into propagation cells $(6.5 \times 6.5 \times 7.5$ $\mathrm{cm})$ filled with a mixture of perlite : commercial medium (Sunshine Mix No. 4; Sun Gro Horticulture, Bellevue, WA) at a $1: 1$ volume ratio and kept on a mist bench until they were well-rooted.

In El Paso, TX, rooted cuttings were transplanted on 19 June to plastic containers $[14.5 \mathrm{~cm}$ diameter, $13.5 \mathrm{~cm}$ height, $2600 \mathrm{~cm}^{3}$ volume (Poly-Tainer No-1P; Nursery Supplies, Orange, CA)] filled with commercial medium (Sunshine Mix No. 4). In Kosciusko, MS, rooted cuttings were transplanted on 12 June to 6 -inch round plastic pots $[15 \mathrm{~cm}$ diameter, $11 \mathrm{~cm}$ height, $1327 \mathrm{~cm}^{3}$ volume (ITML Horticultural Products, Brantford, ON, Canada)] filled with potting mix of pine bark, peatmoss, and sand at a ratio of $5: 4: 1$ by volume. They were irrigated with reverse osmosis water containing $\mathrm{l} \mathrm{g} \cdot \mathrm{L}^{-1}$ of $15 \mathrm{~N}-2.2 \mathrm{P}-12.5 \mathrm{~K}$ [nutrient solution (Peters 15-5-15; Scott-Sierra Horticultural Products Co., Marysville, $\mathrm{OH})]$. The electrical conductivity (EC) and $\mathrm{pH}$ of the nutrient solution were $($ mean $\pm S D) 1.1 \pm 0.1 \mathrm{dS} \cdot \mathrm{m}^{-1}$ and $6.2 \pm 0.2$, respectively. Micronutrients Micromax $^{\circledR}$ Micronutrients; Scott, Marysville, $\mathrm{OH}$ ) were added to each pot at $4 \mathrm{~g} /$ pot. During the experiment, abamectin at $0.08 \mathrm{fl} \mathrm{oz} / \mathrm{gal} \quad[2 \%$ abamectin (AVID ${ }^{\circledR} 25 \%$ EC; Syngenta Crop Protection, Greensboro, NC)] was sprayed as needed to control western flower thrips (Frankliniella occidentalis) and/or melon aphids (Aphis gossypii).

Greenhouse temperature was controlled via pad-and-fan cooling and gas heating systems. In El Paso, $\mathrm{TX}$, the temperature in the greenhouse 

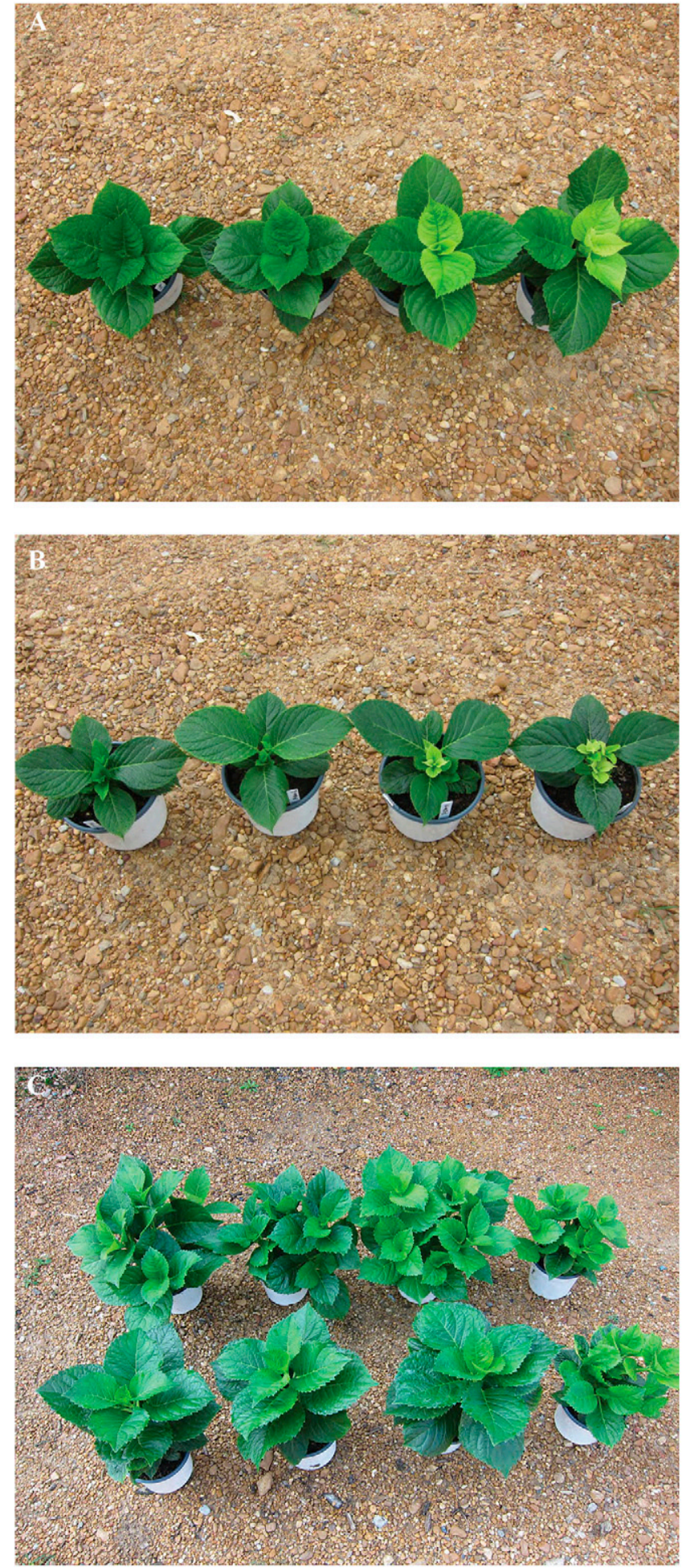

Fig. 1. Photos of 'Merritt's Supreme' bigleaf hydrangea plants from Mississippi taken 2 weeks [(A) unpinched, (B) pinched] and $80 \mathrm{~d}$ after treatment (C). From left to right $(\mathrm{A}, \mathrm{B}$, and $\mathrm{C})$ : application rate of dikegulac sodium at 0 (water), 400, 800, and $1600 \mathrm{mg} \cdot \mathrm{L}^{-1}(\mathrm{ppm})$. Bottom photo: front row: unpinched; back row: pinched.

over the experimental period was measured using $\mathrm{T}$ thermocouples (Omega Engineering, Stamford, CT) connected to a data logger (21X; Campbell Scientific, Logan, UT). Photosynthetic photon flux was recorded with a quantum sensor (Apogee Instruments, Logan, UT). The average air temperatures in the greenhouse were (mean \pm SD) $28.0 \pm 3.4{ }^{\circ} \mathrm{C}$ during the day and $25.4 \pm 2.4{ }^{\circ} \mathrm{C}$ at night from 19 June to 1 Oct. 2012 , and $25.5 \pm$ $3.0^{\circ} \mathrm{C}$ during the day and $22.2 \pm 1.6$ ${ }^{\circ} \mathrm{C}$ at night from 8 Mar. to 7 May 2013. The daily light integral photosynthetically active radiation was $17.9 \pm$ 3.3 and $22.3 \pm 4.0 \mathrm{~mol} \cdot \mathrm{m}^{-2} \cdot \mathrm{d}^{-1}$ from 19 June to 1 Oct. 2012 and from 8 Mar. to 7 May 2013, respectively. In Kosciusko, MS, the day and night temperatures in the greenhouse were $29.0 \pm 3.0^{\circ} \mathrm{C}$ and $21.0 \pm 3.0^{\circ} \mathrm{C}$, respectively, over the period of experiment in 2012 and $23.0 \pm 2.0^{\circ} \mathrm{C}$ and $18.0 \pm 2.0^{\circ} \mathrm{C}$ in 2013 .

On 28 June 2012, 'Merritt's Supreme' bigleaf hydrangea plants were divided into two groups: pinched or unpinched. Pinched plants were hand-pinched to leave two nodes. Two weeks later (i.e., 12 July), dikegulac sodium application was made as a foliar spray to both pinched and unpinched plants at both locations at a rate of 400,800 , or $1600 \mathrm{mg} \cdot \mathrm{L}^{-1}$ according to the IR-4 protocol (IR-4 Project, 2012). There were two control treatments: plants hand-pinched leaving two nodes (pinched) and unpinched plants sprayed with tap water $(\mathrm{EC}=0.8$ $\left.\mathrm{dS} \cdot \mathrm{m}^{-1}, \mathrm{pH}=7.4\right)$. Foliar spray was made to runoff $(\approx 2.5 \mathrm{fl} \mathrm{oz} /$ plant $)$ using a backpack sprayer at both locations.

To determine the blooming responses, plants were moved to a cooler at $4{ }^{\circ} \mathrm{C}$ on 4 Dec. 2012 and moved back to the greenhouse on 8 Mar. 2013 in El Paso, TX, whereas in Kosciusko, MS, plants were moved into a cooler $\left(4.5 \pm 1{ }^{\circ} \mathrm{C}\right)$ in mid-Nov. 2012 and stayed in the cooler for 8 weeks before moving them back to the greenhouse for forcing. Plants were manually defoliated before being placed into the coolers.

Data collection. Initial growth data including plant height (centimeters), two perpendicular widths (centimeters), and the number of branches were recorded. Height was measured from pot rim to the tallest point of the plant. Growth index was calculated as (height + width $1+$ width 2 ) $/ 3$. Phytotoxicity symptoms, reflected as curled leaves, and/or interveinal chlorosis, or bleaching of new growth were evaluated 2 weeks (i.e., 26 July) and 6 weeks after dikegulac sodium application on a 0 to 10 visual 
Table 1. Effect of dikegulac sodium applied as a foliar spray to 'Merritt's Supreme' bigleaf hydrangea on phytotoxicity assessed at 2 and 6 weeks and visual quality assessed at 6 weeks after treatments (WAT) in E1 Paso, TX (TX), and Kosciusko, MS (MS).

\begin{tabular}{|c|c|c|c|c|c|c|c|}
\hline \multirow[b]{3}{*}{ Pinch treatment } & \multirow[b]{3}{*}{ Dikegulac sodium $\left(\mathrm{mg} \cdot \mathrm{L}^{-1}\right)^{\mathrm{z}}$} & \multicolumn{4}{|c|}{ Phytotoxicity (0-10 scale $)^{\mathrm{y}}$} & \multicolumn{2}{|c|}{ Visual quality ( $1-7$ scale $)^{x}$} \\
\hline & & \multicolumn{2}{|c|}{ TX } & \multicolumn{2}{|c|}{ MS } & \multirow{2}{*}{$\frac{\text { TX }}{6 \text { WAT }}$} & \multirow{2}{*}{$\begin{array}{c}\text { MS } \\
\text { 6 WAT }\end{array}$} \\
\hline & & 2 WAT & $6 \mathrm{WAT}$ & $2 \mathrm{WAT}$ & $6 \mathrm{WAT}$ & & \\
\hline Unpinched & 400 & $5.2 \mathrm{c}$ & $0.0 \mathrm{~d}$ & $0.4 \mathrm{c}$ & $0.0 \mathrm{c}$ & $3.9 \mathrm{ab}$ & $4.0 \mathrm{~b}$ \\
\hline Unpinched & 800 & $7.4 \mathrm{~b}$ & $2.0 \mathrm{~b}$ & $0.9 \mathrm{~b}$ & $0.0 \mathrm{c}$ & $3.3 \mathrm{c}$ & $4.0 \mathrm{~b}$ \\
\hline Unpinched & 1,600 & $8.0 \mathrm{ab}$ & $3.8 \mathrm{a}$ & $2.0 \mathrm{a}$ & $1.9 \mathrm{a}$ & $2.8 \mathrm{c}$ & $1.1 \mathrm{~d}$ \\
\hline Pinched & 800 & $7.3 \mathrm{~b}$ & $1.5 \mathrm{bc}$ & $0.6 \mathrm{~b}$ & $0.0 \mathrm{c}$ & $3.4 \mathrm{bc}$ & $6.7 \mathrm{a}$ \\
\hline Pinched & 1,600 & $9.1 \mathrm{a}$ & $4.0 \mathrm{a}$ & $2.0 \mathrm{a}$ & $0.4 \mathrm{~b}$ & $2.8 \mathrm{c}$ & $1.5 \mathrm{c}$ \\
\hline \multicolumn{2}{|l|}{ Pinch } & $\mathrm{NS}^{\mathrm{v}}$ & NS & * & $* * *$ & NS & $* * *$ \\
\hline \multicolumn{2}{|l|}{ Dikegulac sodium } & $* * *$ & $* * *$ & *** & $* * *$ & $* * *$ & $* * *$ \\
\hline
\end{tabular}

${ }^{\mathrm{z}} \mathrm{l} \mathrm{mg} \cdot \mathrm{L}^{-1}=1 \mathrm{ppm}$.

${ }^{y}=$ no phytotoxicity, $10=$ complete kill.

${ }^{\mathrm{x}} \mathrm{l}=$ significantly worse than unpinched without application of dikegulac sodium (untreated), $2=$ moderately worse than untreated, $3=$ slightly worse than untreated, $4=$ no

difference from untreated, $5=$ slightly better than untreated, $6=$ moderately better than untreated, $7=$ significantly better than untreated.

"Means followed with same letters within column are not significantly different among treatments by Tukey's honest significant difference test at $P<0.05$.

${ }^{\mathrm{NS}},{ }^{*},{ }^{* *},{ }^{* *}$ Nonsignificant or significant at $P<0.05,0.01$, or 0.001 , respectively.

scale; 0 representing no phytotoxicity and 10 representing complete kill. Six weeks after treatment (i.e., 22 Aug.), plant height and two perpendicular widths were measured. Visual quality was recorded based on a scale of 1 to 7 , where 1 = significantly worse than unpinched without application of dikegulac sodium (untreated), $2=$ moderately worse than untreated, $3=$ slightly worse than untreated, $4=$ no difference from untreated, $5=$ slightly better than untreated, $6=$ moderately better than untreated, and $7=$ significantly better than untreated. Eighty days after treatment (i.e., 2 Oct.), 10 plants per treatment were harvested in El Paso, TX. Plant height, two perpendicular widths, number of branches, leaf area (square centimeters), and dry weight (gram) were measured. On 7 May 2013, plant height, two perpendicular widths, number of branches and flowers, and diameter of flowers (centimeters) were measured.

EXPERIMENTAL DESIGN AND DATA ANALYSIs. A randomized complete block design with three blocks, eight plants per block, and a total of 24 plants per treatment was used at both locations. A two-way analysis of variance procedure was used to test the effects of pinching and dikegulac sodium on bigleaf hydrangea plant growth. Means separation among treatments was conducted using
Tukey's honest significant difference multiple comparison. Phytotoxicity ratings and visual scores were analyzed by PROC NPARIWAY, which was designed for nonparametric tests. All statistical analyses were performed using SAS (version 9.1.3; SAS Institute, Cary, NC).

\section{Results and discussion}

Two weeks after treatment, all bigleaf hydrangea plants sprayed with dikegulac sodium exhibited phytotoxicity with more pronounced symptoms on plants treated with dikegulac sodium at higher concentrations at both locations (Fig. 1; Table 1). The symptoms included curled new leaves (in some plants), and/or interveinal chlorosis, or bleaching of new growth. Six weeks after treatment, bigleaf hydrangea plants had many fewer phytotoxicity symptoms. There were little or no curled leaves and/or interveinal chlorosis or bleaching of new growth for bigleaf hydrangea plants treated with dikegulac sodium at $400 \mathrm{mg} \cdot \mathrm{L}^{-1}$ at both locations (Table 1). In El Paso, TX, bigleaf hydrangea plants sprayed with dikegulac sodium at 800 or $1600 \mathrm{mg} \cdot \mathrm{L}^{-1}$ experienced more pronounced symptoms with phytotoxicity ratings ranging from 1.5 to 4.0. Unpinched and pinched plants showed similar patterns of phytotoxicity symptoms. In Kosciusko, MS, only bigleaf hydrangea plants treated with dikegulac sodium at $1600 \mathrm{mg} \cdot \mathrm{L}^{-1}$ showed phytotoxicity symptoms with a phytotoxicity rating of 1.9 for unpinched plants and 0.4 for pinched ones. Bigleaf hydrangea plants treated with dikegulac sodium at 0 or $400 \mathrm{mg} \cdot \mathrm{L}^{-1}$ had better visual quality than those with dikegulac sodium at 800 or $1600 \mathrm{mg} \cdot \mathrm{L}^{-1}$ in El Paso, TX. Pinching did not affect whole plants' visual quality. In Kosciusko, MS, bigleaf hydrangea plants treated with dikegulac sodium at 0,400 , or $800 \mathrm{mg} \cdot \mathrm{L}^{-1}$ had better visual quality than those treated at $1600 \mathrm{mg} \cdot \mathrm{L}^{-1}$. Pinched bigleaf hydrangea plants were better than unpinched ones in term of visual quality. Phytotoxicity is a concern with dikegulac sodium application (Sansberro et al., 2006). Similar to previous reports (Cochran et al., 2013), phytotoxicity symptoms of 'Limelight' hardy hydrangea were more pronounced as the concentrations of dikegulac sodium increased. The severity of phytotoxicity symptoms usually decreases over time as seen in this study. In another study, phytotoxicity of Little Lime $^{\mathrm{TM}}$ hardy hydrangea disappeared 16 weeks after treatment (Cochran and Fulcher, 2013).

Six weeks after treatment, both pinching and dikegulac sodium significantly impacted bigleaf hydrangea plant growth at both locations (Table 2). In El Paso, TX, unpinched or pinched bigleaf hydrangea plants were shorter with smaller growth 
index values when they were sprayed with dikegulac sodium at 800 or $1600 \mathrm{mg} \cdot \mathrm{L}^{-1}$. Compared with unpinched plants without dikegulac sodium treatment, height was reduced by $52 \%$ or $58 \%$ and growth index values decreased by $69 \%$ or $62 \%$ when plants were treated with dikegulac sodium at 800 or $1600 \mathrm{mg} \cdot \mathrm{L}^{-1}$, respectively. Pinched plants treated with dikegulac sodium at 800 or $1600 \mathrm{mg} \cdot \mathrm{L}^{-1}$ were $62 \%$ or $90 \%$ shorter with $80 \%$ or $90 \%$ smaller growth index values than pinched plants without dikegulac sodium treatment, respectively. In Kosciusko, MS, unpinched or pinched bigleaf hydrangea plants were similar except those sprayed with dikegulac sodium at $1600 \mathrm{mg} \cdot \mathrm{L}^{-1}$. When unpinched bigleaf hydrangea plants were treated with dikegulac sodium at $1600 \mathrm{mg} \cdot \mathrm{L}^{-1}$, their height and growth index values decreased $82 \%$ and $62 \%$, respectively, compared with unpinched plants without dikegulac sodium treatment. Height of pinched bigleaf hydrangea plants treated with dikegulac sodium at $1600 \mathrm{mg} \cdot \mathrm{L}^{-1}$ decreased $70 \%$ and growth index values decreased $80 \%$, compared with pinched plants without dikegulac sodium treatment. Dikegulac sodium has been documented to effectively control height of Little Lime ${ }^{\mathrm{TM}}$ hardy hydrangea (Cochran and Fulcher, 2013) and liner and finished herbaceous perennial plants (Grossman et al., 2013). The results reported here suggested that dikegulac sodium is an ideal plant growth regulator for height control and for producing compact 'Merritt's Supreme' bigleaf hydrangea.

Eighty days after treatment, both pinching and dikegulac sodium significantly affected bigleaf hydrangea plant height, growth index, and number of branches (Fig. 1; Table 3). Unpinched bigleaf hydrangea plants treated with dikegulac sodium at 800 or $1600 \mathrm{mg} \cdot \mathrm{L}^{-1}$ were $31 \%$ or $21 \%$ shorter than unpinched ones without dikegulac sodium treatments, respectively. Pinched bigleaf hydrangea plants treated with dikegulac sodium at 800 or $1600 \mathrm{mg} \cdot \mathrm{L}^{-1}$ were $19 \%$ or $28 \%$ shorter than pinched ones without dikegulac sodium treatments, respectively. Unpinched bigleaf hydrangea plants treated with dikegulac sodium at 800 or $1600 \mathrm{mg} \cdot \mathrm{L}^{-1}$ had $24 \%$ or $27 \%$ smaller growth index values than unpinched ones without

Table 2. Effect of dikegulac sodium applied as a foliar spray to 'Merritt's Supreme' bigleaf hydrangea on the increases in plant height and growth index assessed at 6 weeks after treatments in El Paso, TX (TX) and Kosciusko, MS (MS).

\begin{tabular}{|c|c|c|c|c|c|}
\hline \multirow[b]{2}{*}{ Pinch treatment } & \multirow{2}{*}{$\begin{array}{c}\text { Dikegulac } \\
\text { sodium }\left(\mathrm{mg} \cdot \mathrm{L}^{-1}\right)^{\mathrm{z}}\end{array}$} & \multicolumn{2}{|c|}{$\mathrm{Ht}(\mathrm{cm})^{\mathrm{z}}$} & \multicolumn{2}{|c|}{ Growth index $(\mathrm{cm})^{\mathrm{z}}$} \\
\hline & & TX & MS & TX & MS \\
\hline Unpinched & 0 & $10.3 \mathrm{a}^{\mathrm{y}}$ & $10.6 \mathrm{a}$ & $10.3 \mathrm{~b}$ & $10.1 \mathrm{ab}$ \\
\hline Unpinched & 400 & $10.6 \mathrm{a}$ & $11.3 \mathrm{a}$ & $8.9 \mathrm{~b}$ & $11.1 \mathrm{a}$ \\
\hline Unpinched & 800 & $5.0 \mathrm{~b}$ & $10.5 \mathrm{a}$ & $3.2 \mathrm{~d}$ & $10.0 \mathrm{ab}$ \\
\hline Unpinched & 1,600 & $4.3 \mathrm{bc}$ & $1.9 \mathrm{~b}$ & $4.0 \mathrm{~d}$ & $3.8 \mathrm{c}$ \\
\hline Pinched & 0 & $5.3 \mathrm{~b}$ & $8.8 \mathrm{a}$ & $13.0 \mathrm{a}$ & $9.0 \mathrm{ab}$ \\
\hline Pinched & 400 & $4.6 \mathrm{bc}$ & $9.6 \mathrm{a}$ & $6.0 \mathrm{c}$ & $9.1 \mathrm{ab}$ \\
\hline Pinched & 800 & $2.0 \mathrm{~cd}$ & $8.1 \mathrm{a}$ & $2.6 \mathrm{de}$ & $8.0 \mathrm{~b}$ \\
\hline Pinched & 1,600 & $0.5 \mathrm{~d}$ & $2.6 \mathrm{~b}$ & $1.4 \mathrm{e}$ & $1.8 \mathrm{c}$ \\
\hline Pinch & & $* * * x$ & ** & * & $* * *$ \\
\hline Dikegulac sodium & & $* * *$ & $* * *$ & $* * *$ & $* * *$ \\
\hline Pinch $\times$ Dikegulac & sodium & NS & NS & $* * *$ & NS \\
\hline
\end{tabular}

${ }^{\mathrm{z}} 1 \mathrm{mg} \cdot \mathrm{L}^{-1}=1 \mathrm{ppm}, 1 \mathrm{~cm}=0.3937$ inch, growth index $=($ height + width + perpendicular width $) / 3$.

y Means followed with same letters within column are not significantly different among treatments by Tukey's honest significant difference test at $P<0.05$.

${ }^{\mathrm{N} S},{ }^{*},{ }^{* *},{ }^{* *}$ Nonsignificant or significant at $P<0.05,0.01$, or 0.001 , respectively

Table 3. Effect of dikegulac sodium applied as a foliar spray to 'Merritt's Supreme' bigleaf hydrangea on plant height $(\mathrm{H})$, growth index (GI), number of branches (NB), leaf area (LA), and dry weight (DW) assessed on 1 Oct. 2012 $(80 \mathrm{~d}$ after treatments) in Texas.

\begin{tabular}{lcccccc}
\hline $\begin{array}{l}\text { Pinch } \\
\text { treatment }\end{array}$ & $\begin{array}{c}\text { Dikegulac } \\
\text { sodium }\left(\mathbf{m g} \cdot \mathbf{L}^{-1}\right)^{\mathrm{z}}\end{array}$ & $\mathbf{H}(\mathbf{c m})^{\mathbf{z}}$ & $\mathbf{G I}(\mathbf{c m})^{\mathbf{z}}$ & $\mathbf{N B}$ & $\mathbf{L A}\left(\mathbf{c m}^{2}\right)^{\mathbf{z}}$ & DW $(\mathbf{g})^{\mathbf{z}}$ \\
\hline Unpinched & 0 & $12.9 \mathrm{bc}^{\mathrm{y}}$ & $13.4 \mathrm{bc}$ & $1.3 \mathrm{bc}$ & $1,633 \mathrm{abc}$ & $22.1 \mathrm{a}$ \\
Unpinched & 400 & $15.1 \mathrm{a}$ & $14.3 \mathrm{bc}$ & $4.8 \mathrm{a}$ & $1,913 \mathrm{ab}$ & $22.9 \mathrm{a}$ \\
Unpinched & 800 & $9.0 \mathrm{e}$ & $10.2 \mathrm{de}$ & $4.9 \mathrm{a}$ & $1,469 \mathrm{bc}$ & $15.4 \mathrm{~b}$ \\
Unpinched & 1,600 & $10.2 \mathrm{de}$ & $9.7 \mathrm{de}$ & $5.5 \mathrm{a}$ & $1,352 \mathrm{c}$ & $12.9 \mathrm{~b}$ \\
Pinched & 0 & $14.5 \mathrm{ab}$ & $18.8 \mathrm{a}$ & $0.7 \mathrm{c}$ & $2,103 \mathrm{a}$ & $24.8 \mathrm{a}$ \\
Pinched & 400 & $14.8 \mathrm{ab}$ & $15.8 \mathrm{~b}$ & $2.0 \mathrm{bc}$ & $1,908 \mathrm{ab}$ & $21.4 \mathrm{a}$ \\
Pinched & 800 & $11.8 \mathrm{~cd}$ & $11.9 \mathrm{~cd}$ & $2.2 \mathrm{~b}$ & $1,552 \mathrm{bc}$ & $14.1 \mathrm{~b}$ \\
Pinched & 1,600 & $10.4 \mathrm{de}$ & $8.5 \mathrm{e}$ & $1.8 \mathrm{bc}$ & $1,177 \mathrm{c}$ & $9.8 \mathrm{~b}$ \\
& & & & & & \\
Pinch & & $* * x$ & $* * *$ & $* * *$ & $\mathrm{NS}$ & $\mathrm{NS}$ \\
Dikegulac sodium & & $* * *$ & $* * *$ & $* * *$ & $* * *$ & $* * *$ \\
Pinch $\times$ Dikegulac sodium & $\mathrm{Ns}$ & $* * *$ & $* * *$ & $*$ & $\mathrm{Ns}$ \\
\hline
\end{tabular}

${ }^{\mathrm{z}} \mathrm{l} \mathrm{mg} \cdot \mathrm{L}^{-1}=1 \mathrm{ppm}, \mathrm{l} \mathrm{cm}=0.3937$ inch, $1 \mathrm{~cm}^{2}=0.1550$ inch $^{2}, \mathrm{l} \mathrm{g}=0.0353 \mathrm{oz}$, growth index $=($ height + width + perpendicular width) $/ 3$

${ }^{y}$ Means followed with same letters within column are not significantly different among treatments by Tukey's honest significant difference test at $P<0.05$.

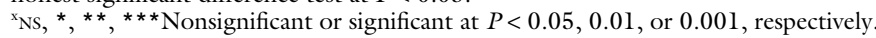

dikegulac sodium treatments, respectively; whereas pinched bigleaf hydrangea plants treated with dikegulac sodium at 400,800 , or $1600 \mathrm{mg} \cdot \mathrm{L}^{-1}$ were $16 \%, 37 \%$, or $55 \%$ smaller than pinched ones without dikegulac sodium treatments, respectively. These data indicate that both unpinched and pinched bigleaf hydrangea plants sprayed with dikegulac sodium at 800 or $1600 \mathrm{mg} \cdot \mathrm{L}^{-1}$ were more compact than those sprayed with 0 or $400 \mathrm{mg} \cdot \mathrm{L}^{-1}$. Dikegulac sodium at all concentrations significantly increased the number of branches of unpinched bigleaf hydrangea plants, while only dikegulac sodium at $800 \mathrm{mg} \cdot \mathrm{L}^{-1}$ increased the number of branches of pinched bigleaf hydrangea plants. In addition, pinching did not increase number of branches. Unpinched bigleaf hydrangea plants treated with dikegulac sodium at 400,800 , or $1600 \mathrm{mg} \cdot \mathrm{L}^{-1}$ had $270 \%, 276 \%$, or $326 \%$, respectively, more branches than unpinched plants without dikegulac sodium treatment, whereas pinched bigleaf hydrangea plants treated with dikegulac sodium at 400,800 , or $1600 \mathrm{mg} \cdot \mathrm{L}^{-1}$ had $186 \%, 214 \%$, or $157 \%$, respectively, more branches than pinched 
Table 4. Effect of dikegulac sodium applied as a foliar spray to 'Merritt's Supreme' bigleaf hydrangea on the increase in plant height, increase in growth index, number of branches, number of flowers, and average diameter of flowers assessed on $7 \mathrm{May}$ 2013 (10 mo. after treatment) in El Paso, TX (TX) and Kosciusko, MS (MS).

\begin{tabular}{|c|c|c|c|c|c|c|}
\hline Pinch treatment & $\begin{array}{c}\text { Dikegulac } \\
\text { sodium }\left(\mathrm{mg} \cdot \mathrm{L}^{-1}\right)^{\mathrm{z}}\end{array}$ & $\mathrm{Ht}(\mathrm{cm})^{\mathrm{z}}$ & $\begin{array}{c}\text { Growth } \\
\text { index }(\mathrm{cm})^{\mathrm{z}}\end{array}$ & Branches (no.) & Flowers $(\text { no })^{\mathrm{y}}$ & Flower diam. $(\mathrm{cm})$ \\
\hline \multicolumn{7}{|c|}{ TX } \\
\hline Unpinched & 0 & $18.5 \mathrm{a}^{\mathrm{x}}$ & $27.5 \mathrm{a}$ & $4.4 \mathrm{abc}$ & $2.4 \mathrm{~b}$ & $13.6 \mathrm{a}$ \\
\hline Unpinched & 400 & $20.7 \mathrm{a}$ & $30.0 \mathrm{a}$ & $6.7 \mathrm{a}$ & $3.7 \mathrm{ab}$ & $13.2 \mathrm{a}$ \\
\hline Unpinched & 800 & $18.0 \mathrm{a}$ & $31.7 \mathrm{a}$ & $6.9 \mathrm{a}$ & $4.4 \mathrm{ab}$ & $12.5 \mathrm{a}$ \\
\hline Unpinched & 1,600 & $17.0 \mathrm{a}$ & $30.8 \mathrm{a}$ & $5.3 \mathrm{ab}$ & $4.0 \mathrm{ab}$ & $13.0 \mathrm{a}$ \\
\hline Pinched & 800 & $18.9 \mathrm{a}$ & $31.3 \mathrm{a}$ & $4.7 \mathrm{abc}$ & $4.8 \mathrm{a}$ & $11.6 \mathrm{a}$ \\
\hline Pinched & 1,600 & $14.6 \mathrm{a}$ & $25.5 \mathrm{a}$ & $3.5 \mathrm{bc}$ & $2.5 \mathrm{~b}$ & $12.0 \mathrm{a}$ \\
\hline \multirow{3}{*}{\multicolumn{2}{|c|}{$\begin{array}{l}\text { Pinch } \\
\text { Dikegulac sodium } \\
\text { Pinch } \times \text { Dikegulac sodium }\end{array}$}} & $\mathrm{NS}^{\mathrm{w}}$ & NS & $* * *$ & NS & NS \\
\hline & & NS & NSS & NS & NS & * \\
\hline & & NS & * & NS & ** & NS \\
\hline Unpinched & 400 & $36.2 \mathrm{a}$ & $31.5 \mathrm{a}$ & $2.3 \mathrm{c}$ & $2.2 \mathrm{c}$ & $21.3 \mathrm{a}$ \\
\hline Unpinched & 800 & $26.3 \mathrm{~b}$ & $31.5 \mathrm{a}$ & $2.3 \mathrm{c}$ & $2.3 \mathrm{c}$ & $20.6 \mathrm{a}$ \\
\hline Unpinched & 1,600 & $28.3 \mathrm{~b}$ & $30.8 \mathrm{a}$ & $7.1 \mathrm{a}$ & $4.3 \mathrm{a}$ & $17.3 \mathrm{~b}$ \\
\hline Pinched & 0 & $34.7 \mathrm{a}$ & $32.9 \mathrm{a}$ & $4.3 \mathrm{~b}$ & $3.2 \mathrm{~b}$ & $21.0 \mathrm{a}$ \\
\hline Pinched & 400 & $34.8 \mathrm{a}$ & $31.1 \mathrm{a}$ & $4.4 \mathrm{~b}$ & $3.2 \mathrm{~b}$ & $19.9 \mathrm{a}$ \\
\hline Pinched & 800 & $35.6 \mathrm{a}$ & $31.6 \mathrm{a}$ & $5.0 \mathrm{~b}$ & $3.1 \mathrm{~b}$ & $20.1 \mathrm{a}$ \\
\hline Pinched & 1,600 & $37.3 \mathrm{a}$ & $32.4 \mathrm{a}$ & $6.6 \mathrm{a}$ & $4.2 \mathrm{a}$ & $17.9 \mathrm{~b}$ \\
\hline \multirow{3}{*}{\multicolumn{2}{|c|}{$\begin{array}{l}\text { Pinch } \\
\text { Dikegulac sodium } \\
\text { Pinch } \times \text { Dikegulac sodium }\end{array}$}} & $\mathrm{NS}^{\mathrm{w}}$ & NS & $* * *$ & $* * *$ & NS \\
\hline & & $* * *$ & NS & $* * *$ & $* * *$ & $* * *$ \\
\hline & & NS & NS & $* * *$ & $* * *$ & NS \\
\hline
\end{tabular}

${ }^{\mathrm{z}} 1 \mathrm{mg} \cdot \mathrm{L}^{-1}=1 \mathrm{ppm}, 1 \mathrm{~cm}=0.3937$ inch, growth index $=($ height + width + perpendicular width $) / 3$.

y Flower was counted only if the diameter $>8 \mathrm{~cm}$.

${ }^{x}$ At each location, means followed with same letters within column are not significantly different among treatments by Tukey's honest significant difference test at $P<0.05$. ${ }^{\mathrm{N} S},{ }^{*},{ }^{* *},{ }^{* *}$ Nonsignificant or significant at $P<0.05,0.01$, or 0.001 , respectively.

ones without dikegulac sodium treatment. These results indicated that dikegulac sodium application could be used as a substitute for hand pinching of bigleaf hydrangea plants to save labor. In a previous study, foliar spray of dikegulac sodium at $1600 \mathrm{mg} \cdot \mathrm{L}^{-1}$ could potentially reduce the amount of pruning required during 'Barbara Karst' bougainvillea production in hanging baskets (Norcini et al., 1993).

Like other PGRs, such as daminozide (Reiss-Bubenheim and Lewis, 1984) and paclobutrazol (Bailey and Whipker, 1998), dikegulac sodium has inhibitory effects on biomass accumulation. Dikegulac sodium significantly affected plant leaf area and shoot dry weight (Table 3). Compared with unpinched bigleaf hydrangea plants without dikegulac sodium treatments, dikegulac sodium at 800 or $1600 \mathrm{mg} \cdot \mathrm{L}^{-1}$ reduced the leaf area of unpinched bigleaf hydrangea plants by $10 \%$ or $17 \%$, respectively. The leaf area of pinched bigleaf hydrangea plants treated with dikegulac sodium at 800 or $1600 \mathrm{mg} \cdot \mathrm{L}^{-1}$ were $26 \%$ or $44 \%$ smaller than pinched ones without dikegulac sodium treatments, respectively. Compared with unpinched bigleaf hydrangea plants without dikegulac sodium treatments, unpinched plants treated with dikegulac sodium at 800 or $1600 \mathrm{mg} \cdot \mathrm{L}^{-1}$ produced $31 \%$ or $42 \%$ less dry biomass, respectively. Pinched plants treated with dikegulac sodium at 800 or $1600 \mathrm{mg} \cdot \mathrm{L}^{-1}$ accumulated $43 \%$ or $60 \%$ less dry biomass than pinched ones without dikegulac sodium treatments, respectively. These results indicated that dikegulac sodium might have significant side effects such as phytotoxicity.
Ten months after treatment, the effects of pinching and dikegulac sodium application on bigleaf hydrangea plants were less obvious (Table 4). In El Paso, TX, no difference existed among treatments in terms of plant height, growth index, and flower size. Foliar application of dikegulac sodium at all tested dosages tended to increase the number of branches and flowers of unpinched plants, but their effects were insignificant. Compared with pinched plants without dikegulac sodium treatment, dikegulac sodium at $400 \mathrm{mg} \cdot \mathrm{L}^{-1}$ increased the number of branches of pinched plants by $150 \%$ but did not increase their number of flowers. Dikegulac sodium at 800 or $1600 \mathrm{mg} \cdot \mathrm{L}^{-1}$ tended to increase the number of branches and flowers of pinched plants, but their effects were insignificant. In Kosciusko, MS, all 
bigleaf hydrangea plants had similar height with the exception of unpinched plants treated with dikegulac sodium at 800 or $1600 \mathrm{mg} \cdot \mathrm{L}^{-1}$. Compared with unpinched plants without dikegulac sodium treatment, dikegulac sodium at 800 or $1600 \mathrm{mg} \cdot \mathrm{L}^{-1}$ reduced unpinched plant height by $24 \%$ or $18 \%$, respectively. Neither pinching nor dikegulac sodium affected the growth index of bigleaf hydrangea plants, but both significantly impacted the number of branches and flowers. For unpinched bigleaf hydrangea plants, those treated with dikegulac sodium at $1600 \mathrm{mg} \cdot \mathrm{L}^{-1}$ had 196\% more branches and 95\% more flowers than those without dikegulac sodium treatment. For pinched bigleaf hydrangea plants, dikegulac sodium at $1600 \mathrm{mg} \cdot \mathrm{L}^{-1}$ increased the number of branches by $53 \%$ and number of flowers by $31 \%$, compared with those without dikegulac sodium treatments. Application of dikegulac sodium at 400 or $800 \mathrm{mg} \cdot \mathrm{L}^{-1}$ on pinched or unpinched plants did not increase the number of branches and flowers. All bigleaf hydrangea plants had similar flower size with the exception of unpinched or pinched plants treated with dikegulac sodium at $1600 \mathrm{mg} \cdot \mathrm{L}^{-1}$. Compared with unpinched or pinched plants without dikegulac sodium treatment, dikegulac sodium at $1600 \mathrm{mg} \cdot \mathrm{L}^{-1} \mathrm{re}-$ duced the flower size of unpinched or pinched plants by $16 \%$ or $15 \%$, respectively. Dikegulac sodium has been reported previously to prevent the development of flower buds of midcentury hybrid lily 'Enchantment' and 'Joan Evans' (Hanks and Menhenett, 1980). In our study, the undesirable side effect of dikegulac sodium on flower number and flower size was minimal.

In conclusion, 'Merritt's Supreme' bigleaf hydrangea plants exhibited severe phytotoxicity 2 weeks after application of dikegulac sodium with more severe symptoms at higher concentrations of dikegulac sodium. The phytotoxicity symptoms became less severe 6 weeks after treatment. The effect of dikegulac sodium on bigleaf hydrangea plant growth, number of branches, and number of flowers depended on location and dosage. In El Paso, TX, dikegulac sodium at 800 or $1600 \mathrm{mg} \cdot \mathrm{L}^{-1}$ inhibited bigleaf hydrangea plant growth at 6 weeks and $80 \mathrm{~d}$ after treatment, and this effect disappeared at 10 months after treatment. Dikegulac sodium at all tested dosages doubled or tripled the number of branches of pinched or unpinched bigleaf hydrangea, respectively, at $80 \mathrm{~d}$ after treatment. At 10 months after treatment, the number of branches and flowers of bigleaf hydrangea plants tended to increase, but was not significant. In Kosciusko, MS, dikegulac sodium at $1600 \mathrm{mg} \cdot \mathrm{L}^{-1}$ reduced the plant growth at 6 weeks after treatment. This treatment increased the number of branches and flowers of unpinched plants by $196 \%$ and by $95 \%$ and pinched plants by $53 \%$ and $31 \%$, respectively, 10 months after treatment. Dikegulac sodium application could be used to increase number of branches and flowers and produce compact 'Merritt's Supreme' bigleaf hydrangea. However, environmental conditions related to locations should be considered in deciding the application concentration of dikegulac sodium.

\section{Literature cited}

Al-Juboory, K.H. and D.J. Williams. 1991. Effects of dikegulac sodium and BA plus GA to enhance lateral branching of golden pothos. Plant Growth Regulat. Soc. Amer. Qrtly. 19:166.

Bailey, D.A. and B.E. Whipker. 1998. Best management practices for plant growth regulators used in floriculture. North Carolina State Coop. Ext. Hort. Info. Lflt. 529. 25 Nov. 2014. <www.ces.ncsu.edu/depts/ hort/floriculture/hils/hil529.html.>.

Carter, J., B.P. Singh, and W. Whitehead. 1996. Dikegulac, but not benzyladenine, enhances the aesthetic quality of boston fern. HortScience 31:978-980.

Cochran, D.R. and A. Fulcher. 2013. Type and rate of plant growth regulator influences vegetative, floral growth, and quality of Little Lime ${ }^{\mathrm{TM}}$ hydrangea. HortTechnology 23:306-311.

Cochran, D.R., A. Fulcher, and G.H. Bi. 2013. Efficacy of dikegulac sodium applied to pruned and unpruned 'Limelight' hydrangea grown at two locations in the southeastern United States. HortTechnology 23:836-842.

Dirr, M.A. 2012. Hydrangeas: Breeding, selection, and marketing. Plant Introductions, Athens, GA.

GIE Media. 2011. Nursery Management, August issue. Plants: What Landscapers Want? I Dec. 2014. <http:// www.nurserymag.com/nm0811-lanscapecontractors-plant-buying-habits.aspx>.

Grossman, M., J. Freeborn, H. Scoggins, and J. Latimer. 2013. Timing of benzyladenine and dikegulac sodium application affects branching of liners and finished herbaceous perennial plants. HortScience 48:875-882.

Hanks, G.R. and R. Menhenett. 1980. An evaluation of new growth retardants on midcentury hybrid lilies. Sci. Hort. 13:349-359.

Hilgers, K.R., C. Haynes, and W.R. Graves. 2005. Chemical height control of containerized seashore mallow. HortTechnology 15:330-332.

IR-4 Project. 2012. Ornamental horticulture protocols: Plant growth regulator protocol 12-013. 25 Nov. 2014. <http://ir4. rutgers.edu/ornamental/ORNDrafts/ 12-013_WoodyBranching.pdf $>$.

Kawabata, O. and R.A. Criley. 1996. Dikegulac sodium spray enhances uniform regrowth of Murraya paniculata (L.) Jack hedge. HortScience 31:244-246.

Norcini, J.G., J.M. McDowell, and J.H. Aldrich. 1993. Dikegulac sodium improves bougainvillea flowering during two production seasons. HortScience 28:119-121.

Reiss-Bubenheim, D. and A.J. Lewis. 1984. Pre-plant treatment of Chrysanthemum $\times$ morifolium with growth retardants. Sci. Hort. 23:279-285.

Sansberro, P., L. Mroginski, and R. Bottini. 2006. Stimulation of lateral branch formation on Ilex paraguariensis (Aquifoliaceae) seedlings. Austral. J. Expt. Agr. 46:707-710.

Sun, Y.P., L.B. Stack, D.L. Zhang, and Z.H.Y. Gu. 2011. Control growth of Euphorbia pulcherrima Willd. ex Klotzsch 'Sonora Jingle' and 'Sonora White' using ethephon. Hort. Environ. Biotechnol. 52:351-356.

Taiz, L. and E. Zeiger. 2010. Plant physiology. 5 th ed. Sinauer, Sunderland, MA.

U.S. Department of Agriculture. 2009. Census of horticultural specialties in 2009. 25 Nov. 2014. <http://www.agcensus. usda.gov/Publications/2007/Online Highlights/Census_of_Horticulture Specialties/hortic_1_023_023.pdf>.

U.S. National Arboretum. 2005. Hydrangea questions and answers. 25 Nov. 2014. <http://www.usna.usda.gov/Gardens/ faqs/hydrangeafaq2.html>.

Wikipedia. 2014. Hydrangea. 25 Nov. 2014. <http://en.wikipedia.org/wiki/ Hydrangea $>$. 